\title{
Opposite Medial Thalamic Unit Responses To Rewarding and Aversive Brain Stimulation
}

\author{
JAMES J. KeEne ${ }^{1}$ \\ Department of Physiology. The Uniacrsity of Michigan, \\ Ann Arbor, Michigan 18104
}

Received September 8, 1972

\begin{abstract}
Medial thalamus receives fibers from both medial forebrain bundle (MFB) and hindbrain and midbrain reticular formation (RET). The MFB and RET stimulations are rewarding and aversive respectively. In 32 unaresthetized cerveau isolé rats, 158 units were recorded. The MFB and RET effects converge on two thirds of the units recorded in the dorsal medial and paracentral nuclei of thalamus and are opposite in the following ways: post-stimulus pattern of unit discharge during $7 \mathrm{~Hz}$ stimulation; slow-wave recruiting with $\mathrm{MFB}$, but not $\mathrm{RET}, 7 \mathrm{~Hz}$ stimulation; and at a "desynchronization" stimulus frequency $(20 \mathrm{~Hz}), \mathrm{MFB}$ elicits decreased unit discharge and RET elicits increased unit discharge, compared to the $7 \mathrm{~Hz}$ rates. In the intralaminar and parafasicular nuclei, post-train $(60 \mathrm{~Hz}, 0.2$ sec) decreases and increases in unit firing lasting seconds are of ten elicited with MFB and RET trains respectively. Stimulation of hypothalamic sites outside MFB did not elicit these MFB effects; parafasicular stimulation did not elicit the RET effects. The MFB effects were not seen in habenula or ventral basal thalamus. Hippocampal, habenular, and ventral medial thalamic units did not show opposite MFB and RET effects. Threshold currents for the opposite $\mathrm{MFB}$ and RET effects are similar to those eliciting self-stimulation and escape respectively in several operated rats tested both hehaviorally and neurophysiologically.
\end{abstract}

\section{INTRODUCTION}

The medial thalamus (15) receives major fiber projections from both the medial forebrain bundle (MFB) (17) and the hindbrain and midbrain reticular formation (RET) $(2,20,28)$. Electrical stimulation of $\mathrm{MFB}$ and RET elicits the highest rates of self-stimulation and escape responding

1 Supported by NIH Grant N-06588. The assistance of Dr. Kenneth L. Casey is gratefully acknowledged. Author's present address: Department of Physiology, University of Puerto Rico Medical Center, Rio Piedras, Puerto Rico. 
respectively (21). These pathways and their anatomical convergence may, therefore, be significant regarding three related neurobehavioral problems: the neural mechanisuss subserving self-stimulation behavior, the transmission of pain-related information into the forebrain, and the integrative mechanisms by which adaptive behavior can be elicited in the presence of both rewarding and aversive stimuli.

It is believed that MFB stimulation is a functional reward with properties similar to conventional rewards, such as food and water, insofar as it can exert similar control over behavior in positive reinforcement paradigms. It is therefore a useful experimental model for the study of reward phenomena.

A number of studies lave attempted to identify efferent pathways essential for MFB self-stimulation by placing lesions destroying the rostral and caudal projections of the MFB (22). Since the lesions did not abolish the behavior, these projections have been judged to be not essential for self-stimulation. In these studies, MFB projections to medial thalamus remained intact and it is possible that this pathway may play a role in self-stimulation behavior.

The following facts are pertinent to a functional analysis of the RETmedial thalamus fibers. First, $\mathrm{A}$-delta and $\mathrm{C}$ fibers in peripheral nerve have been found to be selectively responsive to noxious stimuli $(3,23)$. Second, neurons recorded in nucleus gigantocellularis (NGC) in medullary reticular formation respond selectively to A-delta inputs in cat (6) and to noxious peripheral stimuli in both cat (7) and rat (13). Finally, low-current (100 $\mu \mathrm{a})$ stimulation at the sites from which these neurons were recorded has elicited, in cat (7) and rat (13), escape behavior as well as natural painrelated behavior such as vocalization, crouching or rapid movement, defecation, and urination. These data suggest that the NGC may play a role in pain phenomena.

Other studies have described thalamic unit responses to noxious cutaneous stimulation in rat (1) and monkey (5). The NGC neurons project into the thalamic structures where these nociceptive unit responses were recorded $(2,20,28)$. These projections and the recordings of nociceptive unit responses in NGC suggest that NGC might mediate the thalamic unit responses to peripheral stimulation.

Although pain and reward phenomena have been extensively studied, integrative forebrain mechanisms controlling behavior under conflicting contingencies of reinforcement are poorly understood. Since the medial thalamus receives input from the MFB and RET, which pathways have opposite motivational properties as defined by the behavioral effects of brain stimulation, this neural population might be expected to play a role in integrating information to control behavior in the presence of both 
rewarding and aversive stimuli. Several properties of medial thalamus are perhaps relevant to this speculation.

Units recorded in these thalamic nuclei have been found to be selectively active during volitional behavior in an approach task (18). Bilateral medial thalamic lesions have produced loss of pain sensation in humans (16) and impairment of pain-motivated behavior in cats (19). Such lesions can also result in hypersomnia, loss of consciousness, and coma in human subjects $(4,9)$. Behavioral and electroencephalographic responses often interpreted as increased arousal or attentiveness are elicited with high frequency medial thalamic stimulation; low frequency $(7-10 \mathrm{~Hz})$ stimulation elicits behavioral and slow-wave signs similar to those seen in drowsiness (12). Finally, studies have shown that complex learning and performance of learned behavior are impaired when medial thalamic function is disrupted by lesions (11), stimulation procedures (27), or disease (30). Results such as these present a number of difficult interpretive problems. It is clear, however, that motivation, attention, and learning complex tasks are, in most theoretical treatments of these topics, closely interrelated phenomena. It is noteworthy, then, that such a diverse set of investigations into the neural substrates of these phenomena have assigned a particular importance to medial thalamus.

The present research shows that the effects of MFB and RET stimulations converge on the majority of medial thalamic units tested and that these behaviorally opposite stimulus treatments typically elicit opposite netural responses.

\section{METHODS}

In 32 unanesthetized postcollicular cerveau isolé rats, 158 units have been extracellularly recorded with polished tungsten microelectrodes (Table 1). The 400-g male albino rats were prepared under ether anesthesia. The brain transsection left most of the midbrain including the colliculi intact rostrally. The basic respiratory mechanisms, being controlled caudad to the section, remained functional. No anesthesia was required during the experiments, since the only remaining sensory inputs were visual and olfactory. The section disconnected hypothalamic thermoregulatory mechanisms; rectal temperature was monitored and maintained as necessary. Brain responses and spinal reflexes remained intact for many hours longer than the routine experiments performed.

Ipsilateral stimulation sites were grouped into four categories (Figs. 1 and 2): MFB fiber systcm, hypothalamic sitcs outside MFB (non-MFB), midbrain RET in the region of the NGC-medial thalamus pathway, and parafasicularis (Pf) (Table 1 ).

In cases where chronic brain stimulation was desired for behavioral 


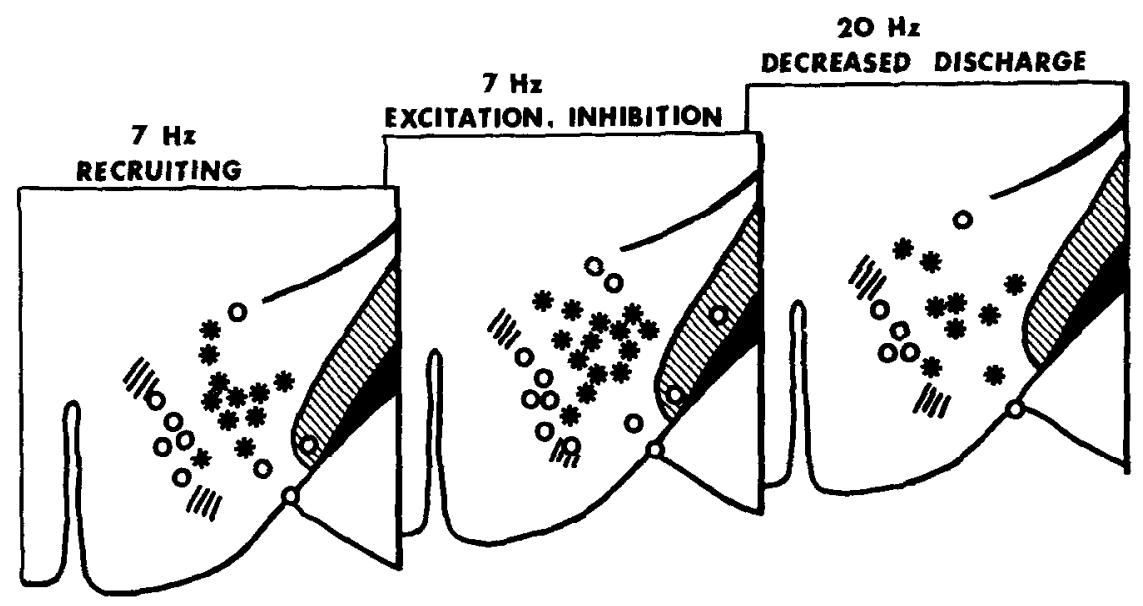

FIG. 1. Hypothalamic sites. For each of three slow-wave or unit responses recorded in the $\mathrm{DM}$ and $\mathrm{PC}$ nuclei, effective (stars) and ineffective (open circles) stimulation sites are mapped in separate drawings. Many sites appear in more than one drawing where two or all three of the response categories were tested in the same animal. These maps suggest that the MFB fiber system is responsible for the medial thalamic (MT) responses described.

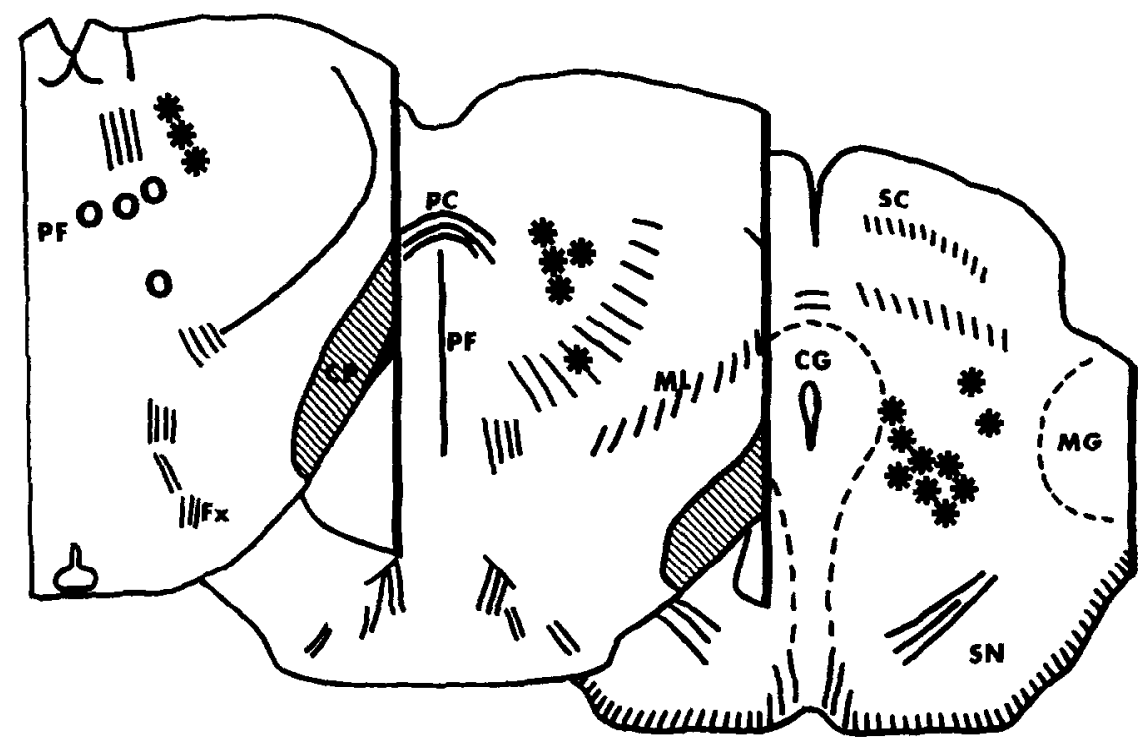

Fig. 2. Midbrain reticular (stars) and parafasicular (open circles) stimulating sites. Reticular and parafasicular stimulations were found to have different effects on $D M$ and $P C$ units. 
TABLE 1

Tabulation of Untes Recurded by Locatimn and Stimelation Sites

\begin{tabular}{|c|c|c|c|c|}
\hline \multirow[b]{2}{*}{ Anatomical structure } & \multicolumn{4}{|c|}{ Stimulus treatments } \\
\hline & $\begin{array}{l}\text { MFB and } \\
\text { RE'T }\end{array}$ & $\begin{array}{c}M F B \text { and } \\
\text { IP }\end{array}$ & $\begin{array}{l}\text { RET and } \\
\text { nonMFB }\end{array}$ & Total \\
\hline \multicolumn{5}{|l|}{ Thalamus } \\
\hline $\begin{array}{l}\text { Dorsal medial and } \\
\text { paracentral nuclei }\end{array}$ & 33 & 20 & 43 & 96 \\
\hline Parafasicularis & 5 & 0 & 0 & 5 \\
\hline $\begin{array}{l}\text { Ventral medial and } \\
\text { reuniens nuclei }\end{array}$ & 6 & 4 & 8 & 18 \\
\hline Ventral basal nucleus & 0 & 12 & 0 & 12 \\
\hline \multicolumn{5}{|l|}{ Other } \\
\hline Habenula & 11 & 0) & 6 & 17 \\
\hline Hippocampus & 4 & 6 & 0 & 10 \\
\hline Sums of units & 59 & 42 & 57 & 158 \\
\hline No. of rats & 15 & 8 & 9 & 32 \\
\hline
\end{tabular}

tests, the rats had stimulation electrodes (twisted pairs of $275 \mu \mathrm{m}$ nichrome wire insulated to the tips) implanted under pentobarbital anesthesia. After recovery, self-stinulation and escape tests were performed as described earlier (13) before acute unit recordings. Daily records were kept of stimulation parameters and response rates until the behavior stabilized in reproducible stimultus-response relationships.

Since frequency is known to be an important stimulus parameter in thalamic slow-wave and unit recordings $(25)$, both synchronizing $(7 \mathrm{~Hz})$ and desynchronizing (20 and $60 \mathrm{~Hz}$ ) frequencies were employed. Stimuli consisted of $0.5 \mathrm{msec}$ monophasic pulses with currents up to $600 \mu \mathrm{d}$ delivered by constant current devices isolated from ground.

Unit responses to successive stimuli were quantified by a poststimulus histogram generator (29), which generates pulses marking the occurrence of isolated action potentials ( $150 \mu \mathrm{v}$ to several $\mathrm{mv}$; stable in amplitude, waveform, and duration) by a level detection system. The pulses were then counted over 60 trials in 10 poststimulus time bins of either 10, 5, or $1 \mathrm{msec}$ duration. Control discharge rates were counted by generating several histograms without stimulation. Units showing changes in control firing rates between different stimulus treatments were excluded from the sample.

$\Lambda$ unit response was defincd as a statistically significant $\left(\chi^{2}, P<0.05\right)$ difference in firing rate between the stimulus train and control periors. 
In addition, for bin-by-bin analysis of poststinulus firing patterns, excitation and inlibition for any particular poststimulus bin was defined. For each unit, the expected variation of the spike counts in the control bins was represented in a $95 \%$ confidence interval computed from the mean control firing rate, the standard deviation of the counts in the control bins, and the appropriate probability level. Excitation for a particular poststimulus bin was then defined as a rate greater than the upper limit of the confidence interval around the control rate. Inhibition or decreased firing in a particular time bin was defined as a count less than the lower limit of the confidence interval. This procedure has the following properties:

First, it is independent of the unil's conirol rate. If, for example, the control rate is high, the spike count in a particular histogram bin must be proportionally higher in order to be categorized as excitation.

Second, this method is sensitive to variations in spontaneous discharge for each unit (e.g., bursts). For units with great variations, the standard deviation of the controls will be greater, specifying a greater upper confidence interval limit. A greater bin spike count would therefore be required to define excitation.

Third, the method is conservative in its definition of inhibition because the lower limit of the confidence intervals is often less than zero for units with low control rates or large variations in spontaneous discharge, or both. This feature is desirable, however, since it reflects the unavoidable problem in defining inhibition with an extracellular recording of a unit with a low baseline firing rate.

Finally, this procedure is systematic, objective, and consistent with visual inspection of the unit response on the oscilloscope display.

Pulses marking spikes, spike rates from electronic integration of the pulses, and slow-wave recordings were documented on moving paper and photographed from an oscilloscope display.

Stimulating and recording electrode tracts were identified through study of $40 \mu \mathrm{m}$ Nissl-stained brain sections. The approximate location of each unit recorded was determined from the known values of the maximal depth of the microelectrode penetration and the depth at which the unit was recorded. The units described below were clearly located within a particular anatomical structure. Terminological debate regarding groupings of medial thalamic nuclei is avoided in this report by referring to specific nuclear structures (Table 1).

\section{RESULTS}

Opposite Effects of $M F B$ and RET Stimulation. In the animals in which both the MFB and RET electrodes were accurately placed (Figs. 1 and 2), it was possible to obtain an estimate of the degree to which MFB and RET 

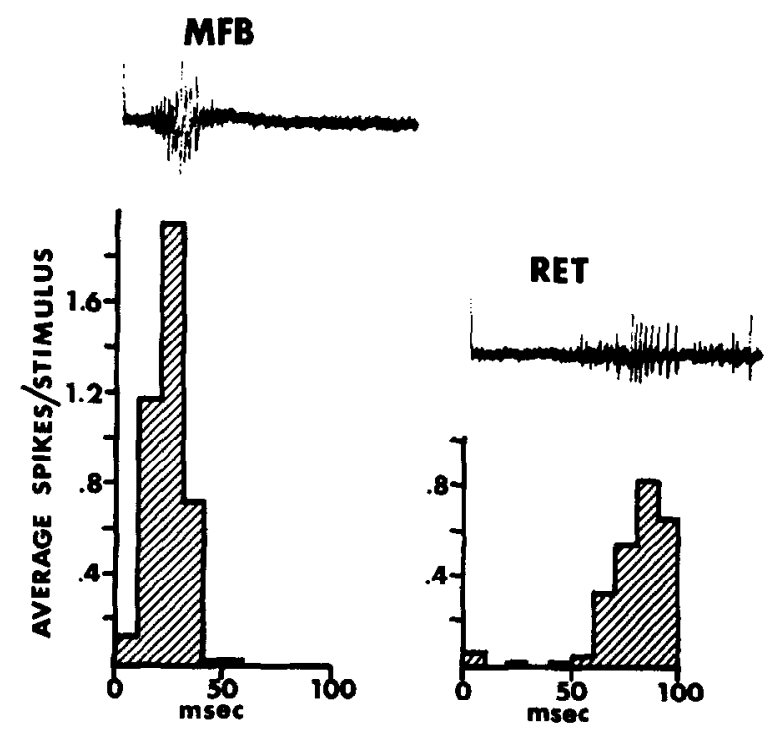

FIg. 3. DM unit with opposite poststimulus patterns during 7-Hz MFB and RET stimulation. The time dimension for the photographs and the histogram illustrations are the same. The histograms represent counts of action potentials of the amplitude of the repetitively firing unit in the RET record. The control rate was 0.1 spikes/ stimulus/bin.

effects converge on single medial thalamic units. In the 33 dorsal medial (DM) and paracentral (PC) units recorded in these animals (Table 1), $66 \%$ were affected by both MFB and RET inputs. Observation of four categories of neural response has revealed that $\mathrm{MFB}$ and RET stimulation typically producc opposite effects in particular nuclei.

Poststimulus discharge pattern during $7-\mathrm{Hz}$ stimulation is illustrated in Fig. 3 which shows a DM unit in which MFB stimuli elicited excitation followed by decreased firing and RET stimuli elicited excitation during the period in which $\mathrm{MFB}$ evoked decreased firing. The responses of the total samples of $\mathrm{DM}$ and $\mathrm{PC}$ units tested with $7-\mathrm{Hz} \mathrm{MFB}$ and RET stimulation are tabulated in Fig. 4. MFB stimuli elicited excitation in 32 of $40(80 \%)$ units at latencies as low as $1.2 \mathrm{msec}$. In over $40 \%$ of the 60 units tested, RET stimuli elicited excitation in the $60-100 \mathrm{msec}$ poststimulus period.

Slow-wave recruiting is elicited by stimulation of the MFB at $7-\mathrm{Hz}$, similar to that seen with low frequency medial thalamic stimulation in cat (26). At DM and PC recording sites, the current thresholds for slow-wave recruiting and the typical pattern of unit driving discussed above were similar, and there appears to be a stimulus-by-stimulus correlation between the number of spikes eliciter and the anplitude of the recruiting slow-wave. All of the sites eliciting these unit responses also evoked recruiting (Fig. 


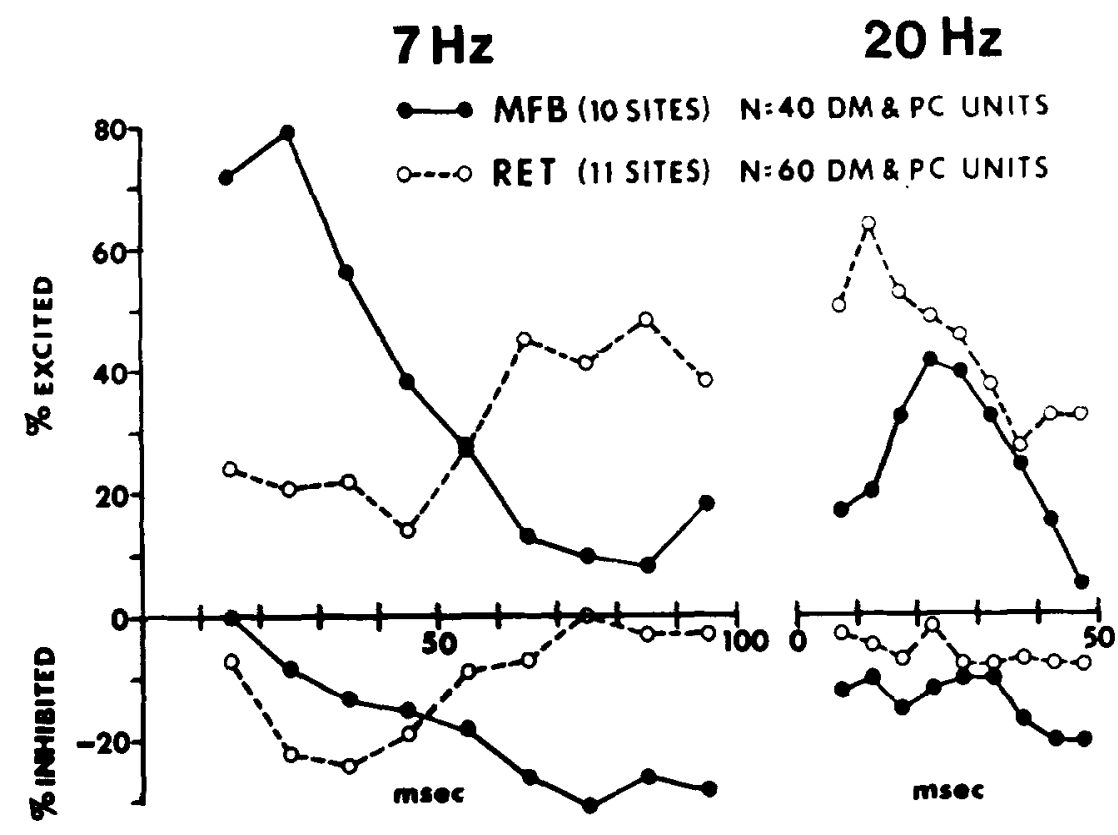

FIG. 4. Tabulation of poststimulus responses of $\mathrm{DM}$ and $\mathrm{PC}$ units to $7-$ and $20-\mathrm{Hz}$ MFB and RET stimulation. The percent of units with responses outside a $95 \%$ confidence interval around the cells' control rates was plotted for each poststimulus bin. With $7-\mathrm{Hz} \mathrm{MFB}$ stimulation, excitation, and then inhibition predominate after the stimulus, $20-\mathrm{Hz}$ stimulation reduces the percentage excited and increases the percentage inhihited in the first $50 \mathrm{msec} ; 7-\mathrm{Hz}$ RET stimuli elicit mixed effects followed by excitation; $20-\mathrm{Hz}$ stimulation increases the percentage excited and decreases the percentage inhibited in the $0-50$ msec poststimulus response.

1). The 7-Hz RET stimuli which elicited a different poststimulus pattern of firing never produced slow-wave recruiting.

Changing the frequency of stimulation from 7 to $20 \mathrm{~Hz}$ produced opposite changes in DM and $\mathrm{PC}$ unit firing rates with $\mathrm{MFB}$ and RET stimulus treatments. With MFB, the mean $20-\mathrm{Hz}$ rate was significantly less than the $7-\mathrm{Hz}$ rate; increasing the RET stimulus frequency to $20 \mathrm{~Hz}$, however, significantly increased the mean spike rate (Figs. 4 and 5). This opposite stimulus frequency effect is also significant in terms of the number of DM and PC units affected (Table 2). Evaluating each unit separately, 36 of the 40 (90\%) units tested with MFB stimulus treatments showed a significant $\left(\chi^{2}, P<0.05\right)$ decrease in spike rate. On the other hand, for 52 of 57 units tested with RET stimulation, the discharge rate with $20-\mathrm{Hz}$ stimulation was greater than with $7-\mathrm{Hz}$ stimulation. For 36 of the 52 (69\%) 11nits, the difference was statistically significant $\left(\chi^{2}, P<0.05\right)$. 


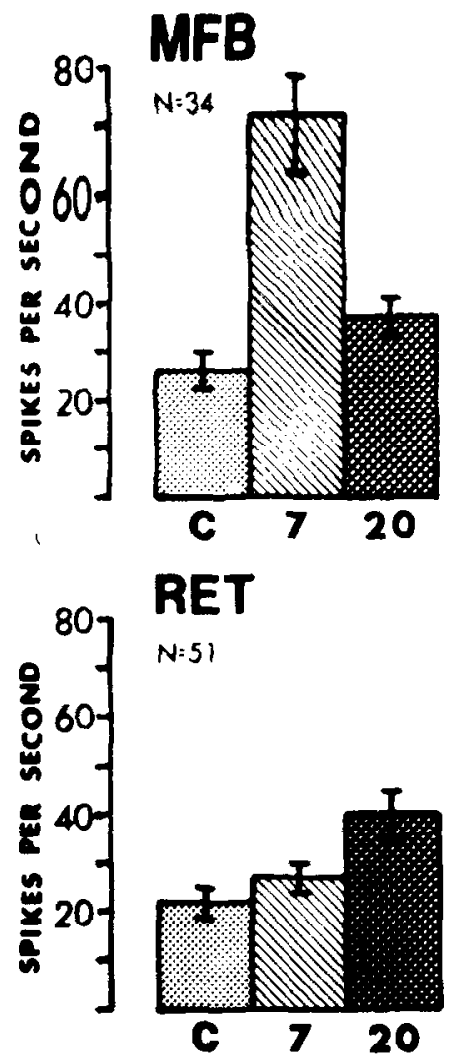

\section{nonMFB}

$N=43$
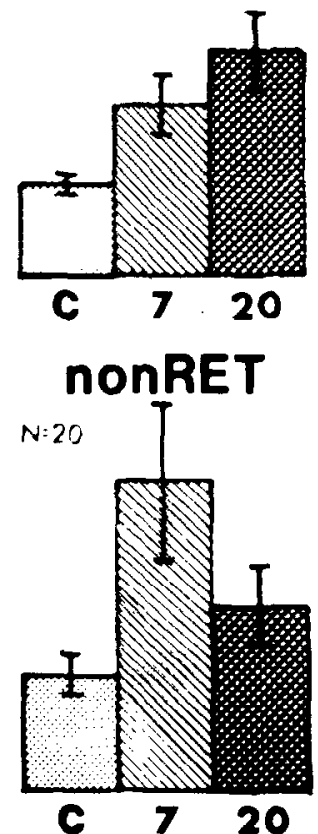

FIG. 5. Effects of stimulation frequency and site on the firing rates of DM and PC units. 7 and $20 \mathrm{~Hz}$ compared to control (C). NonMFB (see text) stimulus treatments produce rate changes similar to those found with RET stimulation. NonRET (Pf) stimulus treatments produce rate changes similar to those elicited by MFB stimulation. The vertical lines indicate SEM's.

TABLE 2

Effect of Increasing Stimulation Frequency on thF SPIKE Rate of DM and PC UNITs ${ }^{n}$

\begin{tabular}{lrrrr}
\hline & MFB & RET & nonMFB & Pf \\
\hline Decreased rate & 39 & 5 & 11 & 13 \\
Significant decrease & 36 & 0 & 5 & 11 \\
Increased rate & 1 & 52 & 28 & 7 \\
Significant increase & 1 & 36 & 24 & 2 \\
Total & 40 & 57 & 39 & 20 \\
\hline
\end{tabular}

a Increasing stimulation frequency from 7 to $20 \mathrm{~Hz}$. 


\section{unit $65-6.65$}

unit 65-67 unit 62-5.8

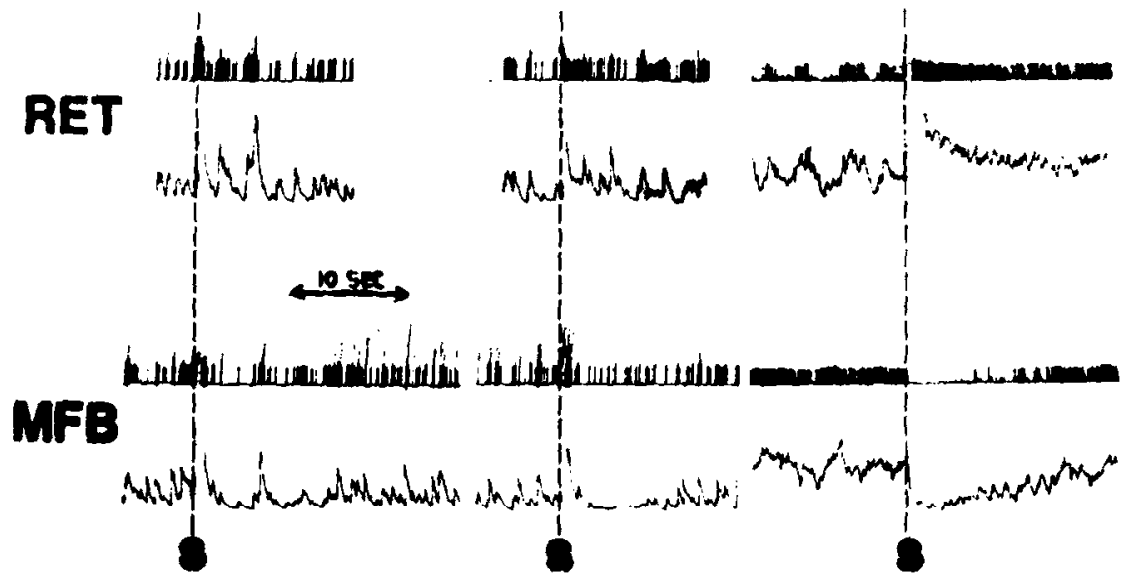

FIG. 6. MFB and RET stimulus trains (S) produce opposite changes in post-train discharge rate. Pulses marking the discharge of a single isolated unit (upper records) and their integration respresenting firing rate (lower records) are shown for two intralaminar units (65-6.65 and 65-6.7) and a parafasicular unit (62-5.8). RE'T stimulus trains elicit prolonged increases in discharge rate. In contrast, similar MFB trains produce prolonged decreases in unit firing.
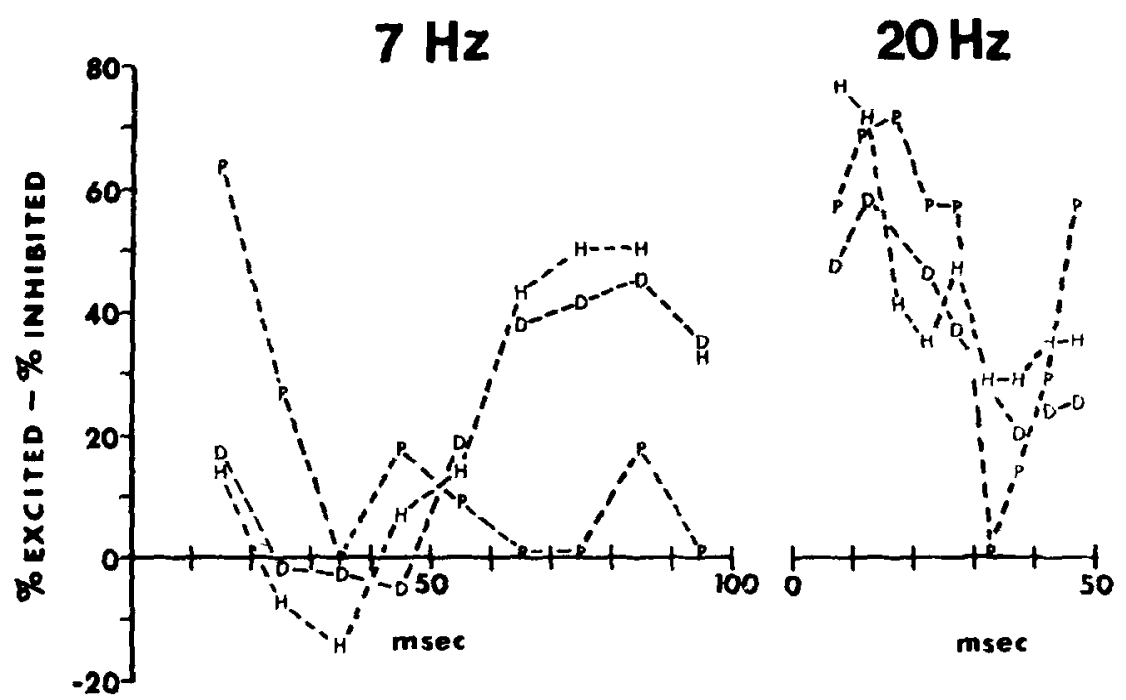

Fig. 7. Effects of 7- and $20-\mathrm{Hz}$ RET stimulation. (D) 60 dorsal medial and paracentral units (derived from Fig. 4); (H) 17 habenular units; (P) 12 parafasicularis units. Each point is the percentage excited minus the percentage inhibited for that time bin and nucleus. $20-\mathrm{Hz}$ RET stimulation excites most of the units in each of these anatomical regions. 


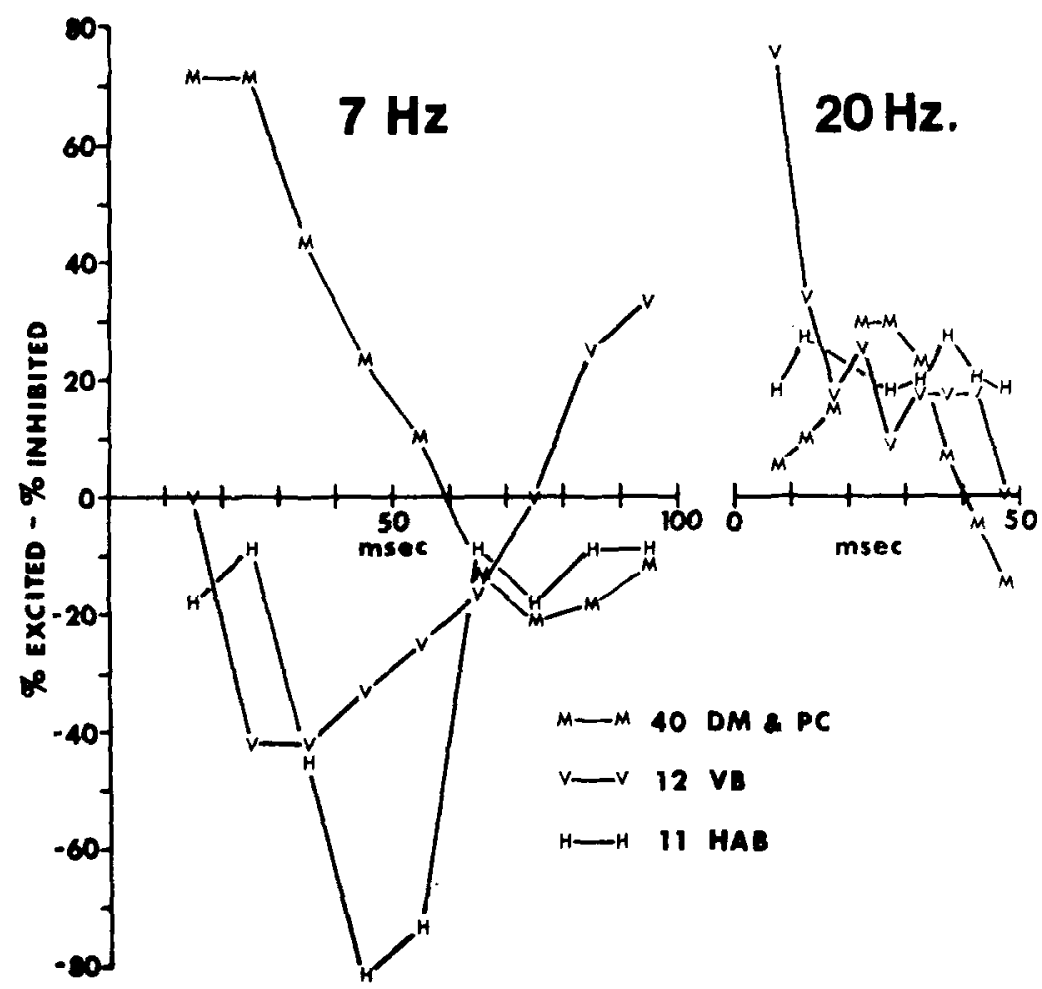

FIG. 8. Differential effects of 7 - and $20-\mathrm{Hz}$ MFB stimulation in diencephalic nuclei. The DM and $P C$ unit respunse curve is derived from Fig. 4. The results from the ventral basal (12 VB units) and habenular (11 HAB units) nuclei are generally opposite to the effects in the DM and PC nuclei.

Post-train changes in unit firing lasting seconds. Data showing increased behavioral effects with increased frequency of brain stimulation (14) suggested that the spike rate differences between $\mathrm{MFB}$ and RE'I stimuli delivered at $20 \mathrm{~Hz}$ might be more related to the behavioral properties of the stimuli. Brief, high frequency stimulus trains $(60 \mathrm{~Hz}, 0.2 \mathrm{sec}$ duration), such as are often used in behavioral tests, were therefore administered. In the PC and Pf nuclei, MFB and RET trains have elicited opposite posttrain changes in unit firing rate lasting several seconds (Fig. 6). Prolonged post-train increases and decreases in unit firing with RET and MFB trains respectively were visible in five out of five $\mathrm{PC}$ and $\mathrm{Pf}$ units tested.

Structures in Which MFB and RET Stimulations Elicit Similar Effects. Midbrain RET stimulation produced responses in 17 habenular units that were similar to those seen in the DM and PC nuclei (Fig. 7). However, the response of 11 of these units tested with MFB stimulation differed markedly from that found in DM and PC units (Fig. 8). The MFB and RET stimulations produced similar changes in firing rate with increased 
stimulus frequency (Figs. 7 and 8). Brief, high frequency RET and MFB stinulus trains $(60 \mathrm{~Hz}, 0.2 \mathrm{sec})$ also produced similar effects in habenula. In four out of four units tested with MFB trains and five out of six units tested with RET trains, the post-train discharge was visibly increased for several seconds.

The MFB and RET stimulus treatments produced similar effects in the ventral medial and reuniens nuclei of thalamus. In two out of four units tested with MFB stimulation and six out of nine units tested with RET stimulation, increasing the stimulus frequency from 7 to $20 \mathrm{~Hz}$ elicited a significant $\left(\chi^{2} P<0.05\right)$ increase in spike rate. A prolonged increase in post-train firing rate was observed in three out of six units tested with MFB trains and in all five units tested with RET trains. Significant, but not opposite, MFB and RET effects were found to converge on four out of four units tested.

Efforts were made to observe prolonged post-train changes in hippocampal unit firing rates. In all ten units tested with MFB trains, and in three out of four units tested with RET trains, no such changes were visible.

The responses to 7 - and $20-\mathrm{Hz}$ MFB stimulation of 12 units in the ventral basal nucleus of thalamus are tabulated in Fig. 8. The 7- $\mathrm{Hz}$ stimuli elicit decreased firing at $20-\mathrm{msec}$ latency, whereas over $70 \%$ of these units are excited at a latency of $5 \mathrm{msec}$ with $20-\mathrm{Hz}$ stimulation. These effects contrast with the effects of similar stimulation on DM and PC units.

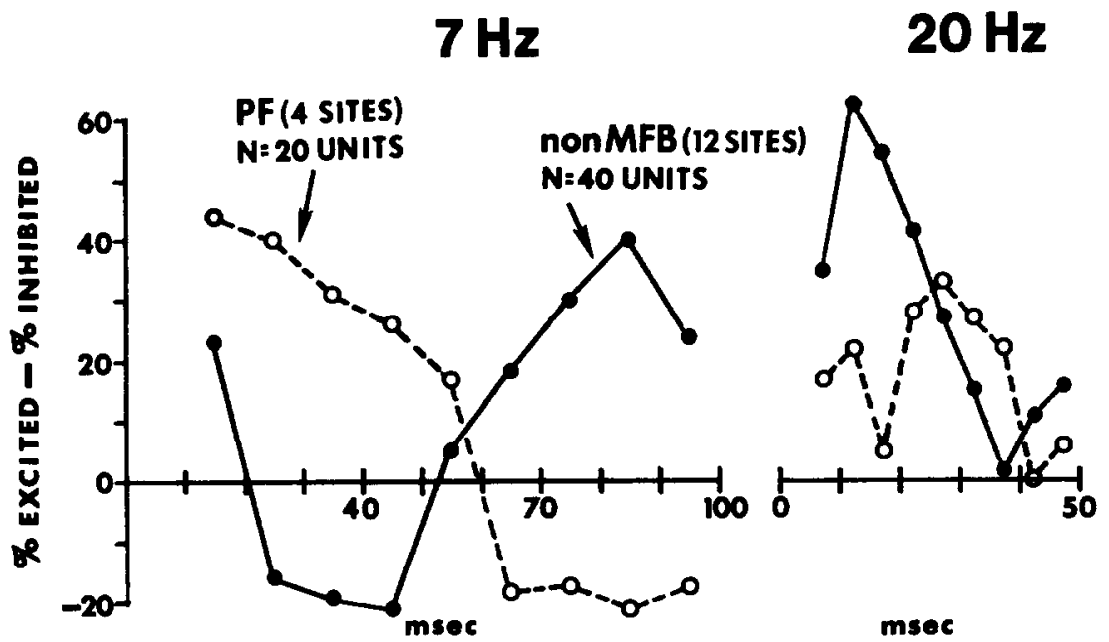

FIg. 9. Poststimulus and stimulus frequency effects on DM and PC units with nonMFB and parafasicular (PF) stimulation; $7-\mathrm{Hz}$ PF stimulation elicits excitation followed by inhibition; $20-\mathrm{Hz}$ stimulation produces less early excitation; $7-\mathrm{Hz}$ nonMFB (see text) stimulation elicits inhibition followed by excitation; $20-\mathrm{Hz}$ stimulation replaces the inhibition with excitation. 
CATHODE TOMADO MFB
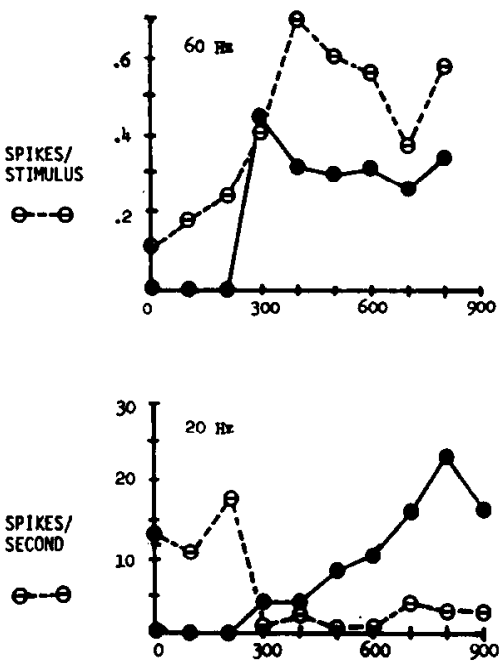

CURRENT (UA)
CATHDOE TONARD MEDIAL HYPOTHALANS
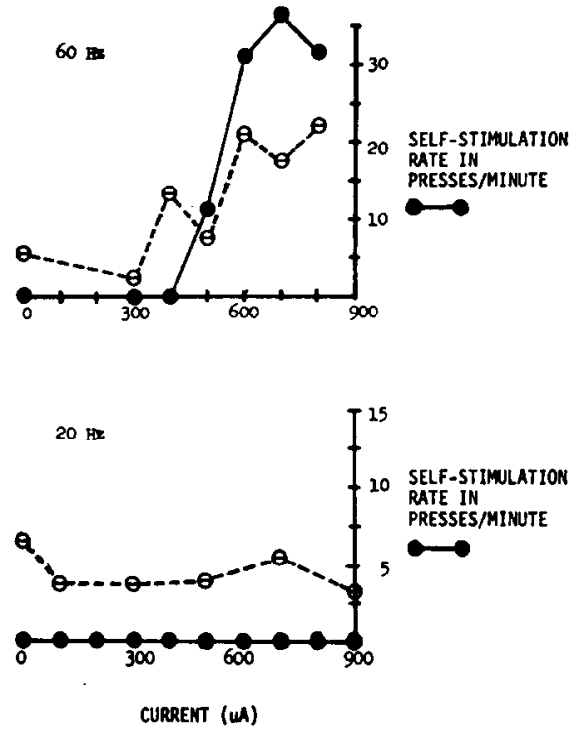

FIG. 10. Correlation between self-stimulation behavior and responses of an intralaminar unit. The behavioral data was obtained from a rat (with electrodes chronically implanted) which was then subjected to acute cerveau isolé unit recording. $60-\mathrm{Hz}$ stimulation: the thresholds and response magnitudes for self-stimulation behavior and unit driving in $0-5 \mathrm{msec}$ periods are closely related. $20-\mathrm{Hz}$ stimulation : the extent of unit inhibition in 5-50 msec poststimulus periods and self-stimulation rate are also closely related. It is not known if either the short-latency driving or the general later inhibition are important in the neural mechanisms subserving self-stimulation.

Parafasicular and Non-MFB Stimulation. In order to define DM and PC unit response properties which may be specific to stimulation of the MFB and RET fiber systems, responses of DM and PC units to stimulation of parafasicularis (Fig. 2) and hypothalamic sites outside MFB (Fig. 1) were tabulated in Fig. 9. In both the poststimulus patterns and stimulus frequency effects, sites outside the MFB fiber system (medial hypothalamus, cerebral peduncle, zona incerta, and meninges) elicited DM and PC unit responses similar to those scen with RET stimulus treatments; the parafasicular effects approximate those elicited by MFB stimulation (Figs. 5 and 9 ; Table 2 ).

Chronic Behavioral Tesls-Acute Unit Recordings. Four of the cervean isolé rats had electrodes chronically implanted for escape testing prior to acute unit recording. In two of these, midbrain RET stimulation elicited both escape behavior and excitation of DM and PC units with the same stimulation parameters. Two other sites (one in parafasicularis; the other not located histologically) failed both to elicit escape and to excite DM and PC units. 
Rat No. 73 had electrodes implanted for self-stimulation tests prior to acute unit recording. Figure 10 shows the stinulus-response curves for both 20 and $60-\mathrm{Hz}$ stimulation and for both polarities of stimulation. The twisted pair was located between medial and lateral hypothalamus. Examination of the two tips of the electrode pair revealed that they were located along a medial-lateral line. The cathode located in the lateral direction toward MFB had a lower threshold for self-stimulation (300 $\mu \mathrm{a})$ for both $60-$ and $20-\mathrm{Hz}$ reward trains $(0.2 \mathrm{sec})$. The cathode located on the medial side had a higher threshold for self-stimulation ( $500 \mu \mathrm{a})$ with $60-\mathrm{Hz}$ trains, and elicited no self-stimulation behavior with $20-\mathrm{Hz}$ trains. The responses of an intralaminar unit near $\mathrm{PC}$ recorded in the acute phase of the experiment are also shown in Fig. 10. The same frequencies and stimulus currents were used to generate poststimulus histograms as were employed in the behavioral tests. For both cathodes, the thresholds and magnitudes of the unit and behavioral responses are remarkably similar.

\section{DISCUSSION}

The three most direct paths from the hypothalamic sites stimulated in this study to medial thalamus are the MFB, the periventricular fibers ascending from medial hypothalamus, and collaterals from internal capsule fibers which then pass by the lateral hypothalamic area in the cerebral peduncle. Analysis of the responses of DM and PC units has revealed three effects of hypothalamic stimulation which almost always occur together: slow-wave recruiting, excitation followed by inhibition with $7-\mathrm{Hz}$ stimulation, and decreased discharge with $20-\mathrm{Hz}$ stimulation (Fig. 1). Sites in or near the MFB or along its projection pathway toward medial thalamus elicit these intercorrelated effects. NonMFB placements in medial hypothalamus, zona incerta, cerebral peduncle, or meninges failed to produce this set of responses. Thus, both the anatomical maps of the stimulation sites and the finding that MFB and nonMFB stimulations have different neural effects, identify MFB stimulation as the effective source of the responses cited. The internuclear differences in MFB-elicited responses revealed in this study may be indicative of related internuclear differences in the effects of MFB activity under normal physiologic conditions.

The diffuse distribution of the endings of MFB and RET fibers in the medial thalamic nuclei suggests that a substantial proportion of the neurons in these nuclei might be affected by these inputs. The estimates of the proportions of these neurons responding to MFB and RET stimuli (Fig. 4) and to both $(66 \%)$ are subject to the following sources of error. $(a)$ Only part of the total population of afferents in these systems were activated by any one stimulation site. (b) A particular unit may be located outside the field of endings activated by a stimulation site. (c) The unit samples 
were obtained on the basis of maintenance of a stable recording and may therefore be unrepresentative of the total population. $(d)$ The cerveau isole operation is likely to alter the excitability of the units recorded. $(e)$ The magnitude of rate changes defined as a response in this study may be quite different from changes which are physiologically significant. With these reservations in mind, it is concluded that the majority of medial thalamic neurons are affected by MFB and RET stimuli. Some of the converging MFB and RET effects may reflect monosynaptic convergence of MFB and RET fibers on single medial thalamic neurons. The lowest response latencies with $\mathrm{MFB}(1.2 \mathrm{msec})$ and $\mathrm{RET}(3 \mathrm{msec})$ stimuli are compatible with the distances, conduction velocities, and times for development of compound postsynaptic potentials that might be involved in monosynaptic or paucisynaptic circuits.

The significance of the convergence of MFB and RET effects on medial thalamic neurons should be considered in the context of the opposite physiological effects of these inputs on DM, PC, and Pf units and the contrasting behavioral effects of MFB and RET stimulation. The RET regions stimulated have elicited escape behavior in two rats in this study and have been preferred sites in other labs requiring reliable elicitation of escape behavior by brain stimulation (21). The rewarding properties of MFB stimulation are also well-established (21). Thus, the RET and MFB stimulations used in this study were probably aversive and rewarding respectively. Other studies of hippocampal slow-wave and unit activity have also failed to show significant differential responses to motivationally opposite stimuli $(10,24)$. The opposite effects elicited in certain medial thalamic units may be systematically related to the motivational properties of MFB and RET stimulation.

The firing rate of DM, PC, and Pf units is not only increased by both peripheral and intracranial aversive stimuli, but is also correlated with the motivating properties of the RET stimuli as measured by behavioral escape rates. In addition, increased firing of these units was also elicited by stimulation of other sites (medial hypothalamus and meninges) which are known to produce escape behavior (21). Further linking aversive brain stimulation with increased unit firing, it was found that $20-\mathrm{Hz}$ stimulation in parafasicularis that did not elicit escape also did not elicit increased firing of these units. These correlations between behavioral and neural responses support the notion that increased discharge in certain medial thalamic units may have a pain-related significance.

Stimulation of the MFB decreased the firing rate in the same population of medial thalamic neurons (Fig. 6). A close correlation between decreased discharge of an intralaminar unit and self-stimulation rates has been observed (Fig. 10). Recordings of Pf units in chronically implanted awake 
rats during self-stimulation have associated rewarding $\mathrm{MFB}$ stimulus trains with prolonged post-train decreased discharge (8) similar to that seen in the PC and Pf nuclei in the present acute experiments. The electrode placements outside the MFB in this study are not associated with self-stimulation (21) and fail to produce the unit responses described with MFB stimulation. These correlations suggest that decreased firing of certain medial thalamic units may be important in the neural mechanisms of reward.

Thus, increased and decreased unit firing in particular medial thalamic nuclei may be motivationally significant responses to RET and MFB inputs respectively. Part of the variation in firing rate of these units over time would therefore be due to the relative activity of the MFB and RET inputs and may play a role in evaluating rewarding and aversive stimuli present at the same time. The general anatomical context of parafasicular and intralaminar neurons may be significant in this respect. These cells also receive polysensory input and are therefore in a position to integrate information regarding rewarding and aversive stimuli with information from other sensory inputs (8). Since these cells project primarily to motor control systems in the forebrain (15), their possible integrative activity resulting from the convergence of motivational and sensory systems may influence the control of behavioral responses to stimulus inputs. These possibilities should be explored in further experiments.

\section{REFERENCES}

1. ANGEL, A. 1964. The effect of peripheral stimulation on units located in the thalamic reticular nuclei. J. Physiol. (London) 171: 42-60.

2. Brodel, A., and G. F. Rossi. 1955. Ascending fibers in brain stem reticular formation of cat. Arch. Neurol. Psychiat. $74: 68-87$.

3. Burgess, P. R., and E. R. Perl. 1967. Myelinated afferent fibres responding specifically to noxious stimulation of the skin. J. Physiol. (London) 190:541562.

4. CaIrns, H. 1952. Disturbances of consciousness with lesions of the brain stem and diencephalon. Brain 75 : 109-146.

5. CASEY, K. L. 1966. Unit analysis of nociceptive mechanisms in the thalamus of the awake squirrel monkey. $J$. Neurophysiol. $29: 727-750$.

6. CASEy, K. L. 1969. Somatic stimuli, spinal pathways, and size of cutaneous fibers influencing unit activity in the medial medullary reticular formation. Exp. Neurol. 25 : 35-56.

7. CASEY, K. L. 1971. Somatosensory responses of bulboreticular units in awake cat: relation to escape producing stimuli. Science 173: 77-80.

8. CASEy, K. L., and J. J. KeEne. 1973. Unit analysis of the effects of motivating stimuli in the awake animal: pain and self-stimulation, pp. In "Brain Unit Activity During Behavior." [M. 1. Phillips, Ed.]. Charles C Thomas, Springfield, Ill.

9. FrENCH, J. D. 1952. Brain lesions associated with prolonged unconsciousness. Arch. Neurol. Psychint. 68 : 727-740. 
10. Fuster, J. M., and A. A. Uyeda. 1971. Reactivity of limbic neurons of the monkey to appetitive and aversive signals. Electrocnccphalogr. Clin. Neurophysiol. $30: 281-293$.

11. Grossman, S. P. 1967. "A Textbook of Physiological Psychology." John Wiley, New York.

12. Jouver, M. 1967. Neurophysiology of the states of sleep. Physiol. Rev. 47: 117177.

13. Keene, J. J, and K. L. Caser. 1970. Excitatory connection from lateral hypothalamic self-stimulation sites to escape sites in medullary reticular formation. Exp. Neurol. 28 : $155-166$.

14. Keesey, R. E. 1962. The relation between pulse frequency, intensity and duration and the rate of responding for intracranial stimulation. I. Comp. Physiol. Psych. $55: 671-678$.

15. KRUPP, P., and M. Monnier. 1966. The unspecific intralaminary modulating system of the thalamus. Int. Rev. Neurobiol. 9: 45-94.

16. Mark, V. H., F. R. Ervin, and P. I. Yakovlev. 1963. Stereotaxic thalamotomy. Arch Neurol. 8 : 528-538.

17. Millhouse, O. E. 1969. A Golgi study of the descending medial forebrain bundle. Brain Res. $15: 341-363$.

18. Mink, W. D., P. J. Best, and J. Olds. 1967. Neurons in paradoxical sleep and motivated behavior. Sicnce 158 : 1335-1337.

19. Mitchell, C. L., and W. W. Kaei.Ber. 1967. Unilateral vs bilateral medial thalamic lesions and reactivity to noxious stimuli. Arch. Neurol. 17: 653-660.

20. Nauta, W. J. H., and H. Kuypers. 1958. Some ascending pathways in the brain stem reticular formation, pp. 3-30. In "Reticular Formation of the Brain." [H. H. Jasper and L. Proctor, Eds.] Little, Brown, and Co., Boston.

21. Olds, J., and M. E. OLds, 1963. Approach-avoidance analysis of rat diencephalon. J. Comp. Neurol. $120: 259-295$.

22. Olds, M. E., and J. Olds. 1969. Effects of lesions in medial forebrain bundle on self-stimulation behavior. Amer. J. Physiol. 217 : 1252-1264.

23. Pert, E. R. 1968. Myelinated afferent fibres innervating the primate skin and their response to noxious stimuli. J. Physiol. (London) 197: 593-615.

24. Pond, F. J., and J. S. Schwartzbaum. 1970. Hippocampal electrical activity evoked by rewarding and aversive brain stimulation in rats. Com. Bchav. Biol. 5 : 89-103.

25. Purpuka, D. P. 1968. Role of synaptic inhibition in synchronization of thalamocortical activity. Progr. Brain Res. 22 : 107-122.

26. Purpura, D. P., and B. Cohen. 1962. Intracellular recording from thalamic neurons during recruiting responses. J. Neurophysiol. 25: 621-635.

27. Rougeul, A., C. Perret, and P. Buser. 1967. Behavioral and electrographic effects of electrical stimulation of the thalamus in the freely moving cat. Electroencephalogr. Clin. Neurophysiol. 23 : 410-428.

28. Scherbel, M. E., and A. B. Scherbel. 1967. Anatomical basis of attention mechanisms in vertebrate brains, pp. 577-602. In "The Neurosciences." [G. C. Quarton, T. Melnechuk, and F. O. Schmitt, Eds.], Rockefeller University Press, New York.

20. Spears, R., G. Smith, and K. L. Casey. 1970. A pulse height discriminator and post-stimulus histogram system using integrated circuits. Physiol. Behav. 5: $1327-1329$.

30. Victor, M., R, D. Adans, and G. H. Coldins, 1971. "The Wernicke-Korsakoff Syndrome." A. F. Davis, Philadelphia. 